\title{
Late Regularization of the Alveolar Ridge in a Patient with a Prosthesis and Special Needs: A Case Report
}

\author{
Wilson Matsumoto ${ }^{1 *}$, Alexandre Elias Trivellato² ${ }^{2}$ Rossana Pereira de Almeida Antunes ${ }^{1}$, \\ Cássio Edvard Sverzut ${ }^{2}$, Guilherme Spagnol ${ }^{2}$, Cristian Sbardelotto ${ }^{3}$, Takami Hirono Hotta ${ }^{1}$ \\ ${ }^{1}$ Department of Dental Materials and Prosthodontics, University of São Paulo, São Paulo, Brazil \\ ${ }^{2}$ Department of Oral and Maxillofacial Surgery and Periodontology, University of São Paulo, São Paulo, Brazil \\ ${ }^{3}$ Department of Dental Materials and Prostheses, University of São Paulo, São Paulo, Brazil \\ Email: *wmatsumoto@forp.usp.br
}

How to cite this paper: Matsumoto, W., Trivellato, A.E., de Almeida Antunes, R.P., Sverzut, C.E., Spagnol, G., Sbardelotto, C. and Hotta, T.H. (2018) Late Regularization of the Alveolar Ridge in a Patient with a Prosthesis and Special Needs: A Case Report. Open Journal of Stomatology, 8, 305-311.

https://doi.org/10.4236/ojst.2018.811028

Received: September 25, 2018

Accepted: October 31, 2018

Published: November 2, 2018

Copyright ( $) 2018$ by authors and Scientific Research Publishing Inc. This work is licensed under the Creative Commons Attribution International License (CC BY 4.0).

http://creativecommons.org/licenses/by/4.0/

(c) (i) Open Access

\begin{abstract}
Residual ridge shape is important for the seating of a prosthesis. The objective of this case report was to illustrate the importance of reduction maneuvers with regard to alveolar bone plates after multiple tooth extractions in patients with prostheses and special needs. In the current patient, there were difficulties during the adaptation and fitting of the upper denture. Bilateral bone resection was indicated in the molar buccal region. Late regularization of the alveolar ridge, which was accomplished in hospital, was necessary for the preparation, adaptation, and use of the denture. The general and systemic health care of the patient should be monitored throughout surgical intervention. Though tooth extractions are common procedures, particular attention is required to ensure adequate alveolar ridge remodeling that is conducive to oral rehabilitation.
\end{abstract}

\section{Keywords}

Alveolar Ridge Adaptation, Denture, Exostoses

\section{Introduction}

When the extraction of teeth is required, the clinician must plan the surgical procedure aiming to preserve the remaining alveolar bone [1] [2] [3] while maintaining due consideration of the alveolar curettage, alveoloplasty, and bone plate repositioning. These procedures are important to prevent substantial bone resorption and the undue retention of bone tissue that could result in bone ex- 
ostosis and interfere with the final results of alveolar bone remodeling, and therefore compromise the success of prosthetic rehabilitation. As well as these specific care procedures, the patient's general health condition must be considered.

Some patients have special needs resulting from acquired or congenital systemic problems or invasive procedures. These types of patients require particular attention when surgical procedures are necessary. Even if the interventions required are considered simple, they must be well planned and executed. The objective of this case report was to describe the consequences of the presence of bone projections on the alveolar ridge in a patient with a complete denture and special needs.

\section{Case Report}

The publication of this case report was approved by the Ethics Committee on Human Research of the Ribeirão Preto Dental School, University of São Paulo, Brazil, and all treatment was conducted in accordance with ethical standards and the Declaration of Helsinki. The patient was a 46-year-old woman who attended the Dental School of Ribeirão Preto at the University of São Paulo for evaluation and prosthetic treatment. She was a denture user due to multiple extractions performed 4 months prior, at a different hospital. Functional instability and frequent bilateral ulcerations in molar regions were reported during the clinical examination. Prominent bony oral projections from the molar region to the maxillary tuberosity similar to exostosis were observed (Figure 1). An impression of the upper arch was acquired with alginate, to facilitate the generation of an initial model (Figure 2).

The patient was referred for surgery, but due to her medical history of systemic lupus erythematosus, hemolytic anemia, and venous thrombosis, and the use of chloroquine diphosphate, cilostazol (Cebralat), benfotiamine (Milgam$\mathrm{ma}$ ), and oral anticoagulant therapy (warfarin-derived 4-hidroxicoumarin; Marevan), the surgery was scheduled to be performed in the hospital. The replacement of Marevan with low molecular weight heparin (LMWH), subcutaneously every 12 hours, was performed 3 days before the surgery. A coagulation examination was performed 1 day before the surgery via the international normalized ratio (INR) method, which standardizes the prothrombin time analysis, and the result was 1.47. Anticoagulation with LMWH was maintained for 3 more days. With the Marevam suspension, LMWH maintained the anticoagulation. The procedure was conducted in hospital due to the risk of bleeding, and thereafter Marevan was reinstated.

The patient was admitted to the hospital on the day of the surgery and taken to the operating room. The nasotracheal intubation was achieved and the general anesthesia was maintained combining intravenous and inhaled agents. After the intra and extraoral antisepsis applying a topical solution of polyvinylpyrrolidone $10 \%$, the patient was covered with sterile drapes leaving only the face uncovered. 


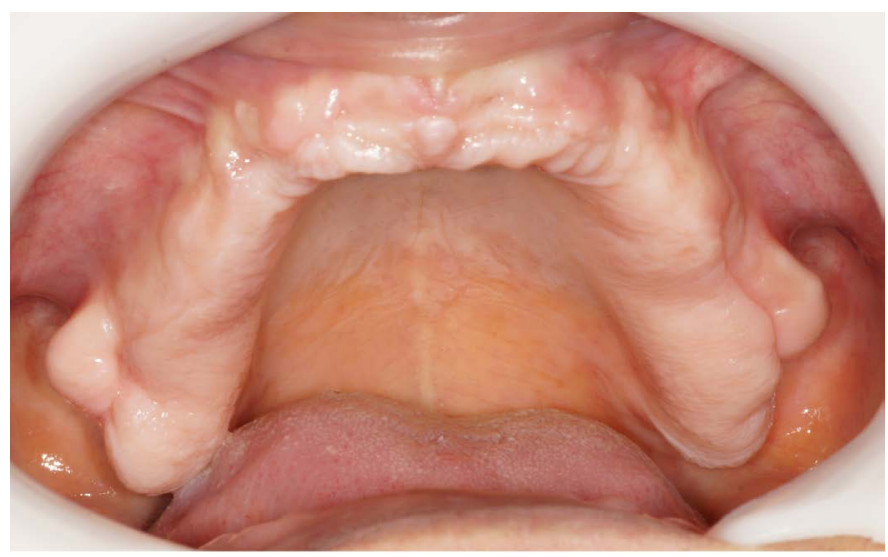

Figure 1. Initial occlusal view of the maxillary arch showing bucalexostoses in the molar regions.

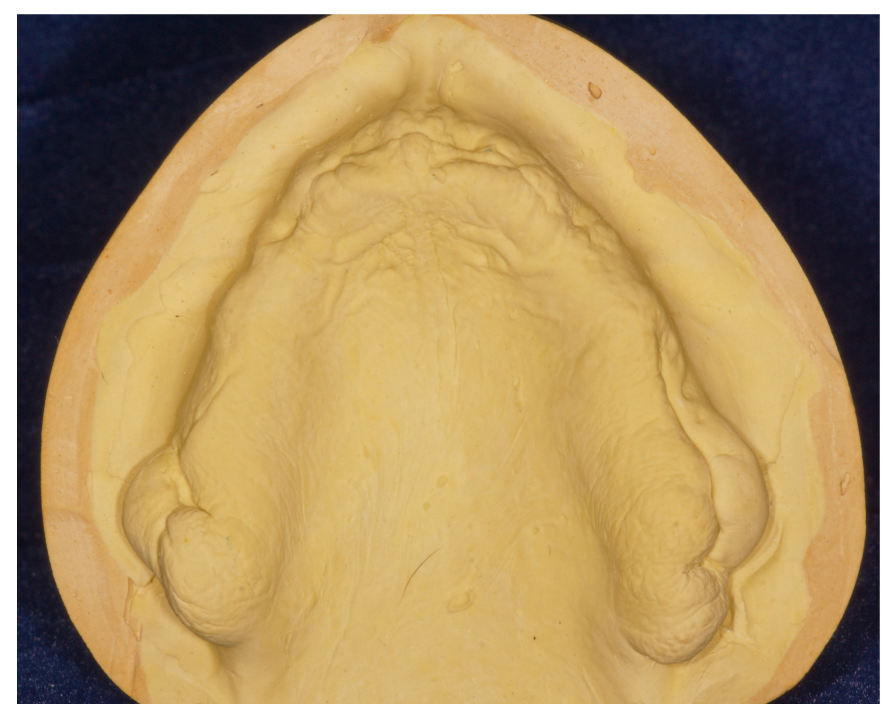

Figure 2. Initial posterior anterior view of the maxillary gypsum cast showing bucalexostoses in the molar regions.

At the molar region of the right side, a linear incision was made over the crest of the alveolar residual ridge applying a surgical blade \#15 and a mucoperiosteal flap was elevated exposing all the hyperplasic bone tissue. To achieve an adequate shape of the alveolar residual ridge, an egg head carbide bur was applied under intense irrigation with sterile saline solution removing the excessive bone. Afterward, a bone file \#11 was applied to finish the osteoplasty and an intense irrigation with sterile saline solution was performed in order to remove the bone debris. The wound was sutured in a continuous fashion applying a 4 - 0 absorbable thread (poliglactine 910). The same procedure was performed on the left side of the molar region. The postoperative period was uneventful.

Forty days after the surgery, the patient returned to commencerehabilitation procedures. The main support area of the denture exhibited proper conformation and no irregularities (Figure 3). Impressions of the maxillary and mandibular arches were made with alginate, and gypsum casts were generated. Custom 
acrylic resin trays were made to facilitate the generation of a functional impression. The functional impression was made with low fusion compound and zinc oxide and eugenol paste to generate the working casts. Occlusion rims were made on the working casts, to enable registration of the intermaxillary relationship and mounting of the casts in a semi-adjustable articulator. After clinical approval of tooth mounting, dentures were installed and adjusted (Figure 4).

According to the patient report and clinical observation after resection of bone protuberance, the use of the prosthesis caused no more injuries to the mucosa and there was a better fit and containment, promoting more functional chewing, deglutition, and speaking.

\section{Discussion}

Systemic lupus erythematosus is a chronic inflammatory autoimmune disease of unknown etiology. Epidemiological studies have shown that women are much more affected than men, with a range varying from 6 to 10 women for every man stricken [4]. It may lead to complications including immune system alterations, rheumatoid arthritis, kidney failure, central nervous system disorders, and blood

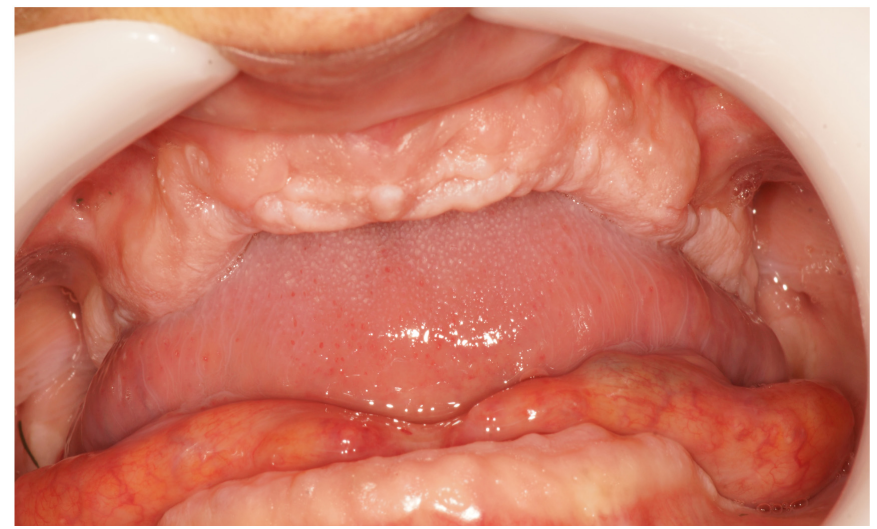

Figure 3. Occlusal view of the maxillary arch after surgery, without the exostoses.

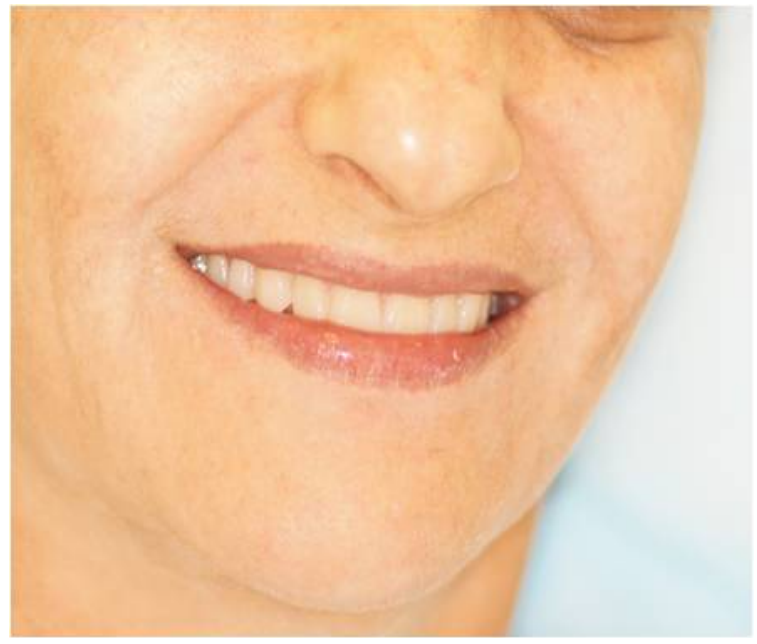

Figure 4. Frontal view of the patient's smile with the new complete dentures. 
disorders such as hemolytic anemia, and requires attention during surgical dental procedures. A lot of the medications used to treat the disease lead to immunosuppression, predisposing to infection. The medication dose must be adjusted to prevent renal failure, and the use of non-steroidal anti-inflammatories must be avoided [5]. In the present case, the use of anticoagulants due to venous thrombosis was an added complication.

Vitamin $\mathrm{K}$ inhibitors protect the patient from thromboembolic events, but they predispose them to bleeding during or after surgery. For this reason, 3 days before the surgery oral therapy with warfarin was replaced with LMWH, which has a short half-life of 2 - 4 hours. The coagulation test (prothrombin time) was performed because it evaluates the extrinsic and common coagulation pathway, and dependent factors of vitamin $\mathrm{K}$. Although the INR values for dental procedures described in the literature are variable [6] [7], it is recommended that 24 hours before dental surgery the test result should be below 2.0. In the present case the test result of 1.47 was close to normal, thus it was not indicative of a risk of bleeding complications such as undue anemia or bruising in the operated area.

Some types of bony projections can remain in the oral cavity without being noticed by the patient, and are only noticed during the course of routine clinical examination or when oral rehabilitation becomes necessary. Bone removal can be indicated in order to preclude injury to the mucosal tissue [8] [9] when total or partial removable dentures are planned, or to improve denture stability and prevent problems during swallowing and speech. As the current patient had used an upper denture supported by bilateral buccalbony projections, she was exposed to functional disorders, pain, and insecurity with regard to using her new denture.

The length and shape of some bony projections necessitate the use of a guide during surgery [10], in order to achieve a uniform edge that is conducive to a well-fitting prosthesis. A surgical guide was not used in the present case because the bone protrusions had well defined limits and locations, they were classified as moderate [11], and they were not interfering with the conformation of the prosthesis-bearing area.

Before any surgical intervention, an adequate treatment plan should be made and the patient must be informed and agree with the treatment. In the current case wherein the patient had special needs, a second surgery was conducted in a hospital in which all the associated risks and cost could have been avoided if due attention had initially been paid to predicting the outcome with regard to the edge of the remodeling process, and the subsequent need to adapt the anatomical conditions for prosthetic rehabilitation.

The compression of socket walls after closed extraction is one type of alveoloplasty procedure that is widely used with the intention of causing the tooth socket to return to a primitive form, via digital compression of the cortical bone [12], but it can also be used during osteotomies and to accommodate the con- 
tours of bone plates. When performed improperly, these procedures can promote pronounced resorption of bone tissue. In the present patient, the initial surgical maneuvers performed were not sufficient because relatively substantial bony projections remained in regions that compromised the success of rehabilitation.

According to Kivovics et al. [13], the traumatic ulcer lesions, red or white, are common after complete denture has been delivered and they are caused by improperly fitting dentures.

The etiological factors of the traumatic lesions seem to be related to denture factors, including superficial irregularities, overextension, and occlusal instability; to local factors such as exostosis, residual ridge resorption, and lack of submucosal supportive tissues; and to systemic factors, chiefly diabetes and immunologic disorders [14]. In this clinical case, the mucosal tissues that covered the exostoses appeared injured and painful which prevented the functional activities be accomplished.

To minimize or eliminate the oral mucosal lesions, dental treatment should have the objective to produce improvements in denture stability [14] as in the present case, the surgical removal of exostoses was chosen to adequate the area of support for the new denture.

\section{Conclusion}

Though tooth extractions are common procedures, they require meticulous attention by the surgeon in order to achieve adequate remodeling of the alveolar ridge that facilitates good oral rehabilitation, especially in cases where the surgery is associated with specific risks in a particular patient.

\section{Conflicts of Interest}

The authors declare no conflicts of interest regarding the publication of this paper.

\section{References}

[1] Atwood, D.A. and Coy, W.A. (1971) Clinical, Cephalometric and Densitometric Study of Reduction of Residual Ridges. The Journal of Prosthetic Dentistry, 26, 280-295. https://doi.org/10.1016/0022-3913(71)90070-9

[2] Bartee, B.K. (2001) Extraction Site Reconstruction for Alveolar Ridge Preservation. Part 1: Rationale and Materials Selection. The Journal of Oral Implantology, 27, 187-193. https://doi.org/10.1563/1548-1336(2001)027<0187:ESRFAR>2.3.CO;2

[3] Irinakis, T. (2006) Rationale for Socket Preservation After Extraction of a Single-Rooted Tooth When Planning for Future Implant Placement. Journal of the Canadian Dental Association, 72, 917-922.

[4] de Araújo Navas, E.A.F., Sato, E.I., Pereira, D.F.A., Back-Brito, G.N., Ishikawa, J.A., Jorge, A.O.C., Brighenti, F.L. and Koga-Ito, C.Y. (2012) Oral Microbial Colonization in Patients with Systemic Lupus Erythematous: Correlation with Treatment and Disease Activity. Lupus, 21, 969-977.

https://doi.org/10.1177/0961203312443420 
[5] Louis, P.J. and Fernandes, R. (2001) Review of Systemic Lupus Erythematosus. Oral Surgery, Oral Medicine, Oral Pathology, Oral Radiology, and Endodontics, 91, 512-516. https://doi.org/10.1067/moe.2001.114153

[6] Aframian, D.J., Lalla, R.V. and Peterson, D.E. (2007) Management of Dental Patients Taking Common Hemostais-Altering Medications. Oral Surgery, Oral Medicine, Oral Pathology, Oral Radiology, and Endodontics, 103, e1-e11.

[7] Kammerer, P.W., Frerich, B., Liese, J., Schiegnitz, E. and Al-Nawas, B. (2015) Oral Surgery during Therapy with Anticoagulants-A Systematic Review. Clinical Oral Investigations, 19, 171-180. https://doi.org/10.1007/s00784-014-1366-3

[8] Pack, A.R., Gaudie, W.M. and Jennings, A.M. (1991) Bony Exostosis as a Sequelato Free Gingival Grafting: Two Case Reports. Journal of Periodontology, 62, 269-271. https://doi.org/10.1902/jop.1991.62.4.269

[9] Chaudhry, S.I., Tappuni, A.R. and Challacombe, S.J. (2000) Multiple Maxillary and Mandibular Exostoses Associated with Multiple Dermatofibromas. A Case Report. Oral Surgery, Oral Medicine, Oral Pathology, Oral Radiology, and Endodontics, 89, 319-322. https://doi.org/10.1016/S1079-2104(00)70096-5

[10] Jung, S.R. and Bashutski, J.D. (2010) A Bone-Level Stabilized Surgical Template to Manage Enlarged Nonrestorable Maxillary Tuberosities. The Journal of Prosthetic Dentistry, 103, 319-320. https://doi.org/10.1016/S0022-3913(10)60066-2

[11] Pechenkina, E.A. and Benfer Jr., R.A. (2002) The Role of Occlusal Stress and Gingival Infection in the Formation of Exostoseson Mandible and Maxilla from Neolithic China. HOMO, 53, 112-130. https://doi.org/10.1078/0018-442X-00040

[12] Parvez, A., Malik, M.A. and Sheikh, M.A. (2013) Incidence of Alveoloplasty and Its Indications-Protocol to Reduce the Incidence. Pakistan Oral \& Dental Journal, 33, 236-239

[13] Kivovics, P., Jáhn, M., Borbély, J. and Márton, K. (2007) Frequency and Location of Traumatic Ulcerations Following Placement of Complete Dentures. International Journal of Prosthodontics, 20, 397-401.

[14] Martori, E., Ayuso-Montero, R., Martinez-Gomis, J. and Viñas, M. (2014) Risk Factors for Denture-Related Oral Mucosal Lesions in a Geriatric Population. The Journal of Prosthetic Dentistry, 111, 273-279. https://doi.org/10.1016/j.prosdent.2013.07.015 